\title{
Caracterização pós-colheita de frutos de maracujá-roxo cultivados em sistema convencional e orgânico
}

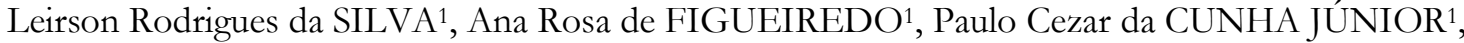 \\ Maria Ivone Martins Jacintho BARBOSA ${ }^{1}$, Milena Maria Tomaz de OLIVEIRA², \\ Raul Castro Carriello ROSA ${ }^{3}$, Lilia Aparecida Salgado de MORAIS 3 \\ ${ }^{1}$ Universidade Federal Rural do Rio de Janeiro, Seropédica, RJ, Brasil. \\ ${ }^{2}$ Universidade Ben-Gurion de Negev, Sde Boker, Israel. \\ ${ }^{3}$ Embrapa Agrobiologia, Seropédica, RJ, Brasil. \\ *E-mail: rodriguesleirson@yahoo.com.br \\ (ORCID: 0000-0001-7216-2293; 0000-0003-3536-5512; 0000-0002-9414-4915; 0000-0002-9624-9139; \\ 0000-0001-7345-1003; 0000-0002-2093-7752; 0000-0002-5382-6485)
}

Recebido em 08/10/2021; Aceito em 02/12/2021; Publicado em 17/12/2021.

\begin{abstract}
RESUMO: Cultivares de maracujá-roxo desenvolvidas regionalmente podem contribuir para o aumento da produção de frutos de qualidade atendendo às necessidades do mercado. Objetivou-se avaliar os frutos de maracujá-roxo, obtidos em sistemas de cultivo convencional e orgânico. Os frutos foram colhidos em estágio de maturação comercial (casca totalmente roxa) e analisados quanto à caracterização físico-química, centesimal, compostos bioativos e capacidade antioxidante. Os frutos analisados apresentaram maiores valores médios para o rendimento de polpa e sólidos solúveis em sistema convencional, que foram de 49,04\% e 13,86 ${ }^{\circ} \mathrm{Brix}$. O valor calórico energético foi de 45,95 kcal/100 g em sistema orgânico, indicando que o consumo do fruto "maduro" pode ser realizado a fim de suprir as necessidades energéticas diárias. As polpas dos frutos de maracujá-roxo estudados demonstraram serem fontes importantes de componentes bioativos, com respaldo aos elevados valores médios para ácido ascórbico e polifenóis extraíveis totais em ambos os sistemas de cultivo.
\end{abstract}

Palavras-chave: Passiflora edulis Sims; manejo; sólidos solúveis; compostos fenólicos.

\section{Postharvest characterization of purple passion fruits cultivated in conventional and organic system}

\begin{abstract}
Regionally developed purple passion fruit cultivars can contribute to the increase in the production of quality fruits, meeting marked needs. The objective of this study was to evaluate the purple passion fruits, obtained in conventional and organic cultivation systems. The fruits were harvested at the stage of commercial maturation (totally purple peel) and analyzed for characterization of the physicochemical, centesimal composition, bioactive compounds and antioxidant capacity. The analyzed fruits showed higher average values for pulp yield and soluble solids in a conventional system, which were $49.04 \%$ and $13.86^{\circ}$ Brix. The caloric energetic value was $45.95 \mathrm{kcal} / 100 \mathrm{~g}$ in organic system, indicating that the consumption of the "ripe" fruit can be carried out in order to meet the daily energy needs. The pulps of the purple passion fruit studied proved to be important sources of bioactive components, supported by the high mean values for ascorbic acid and total extractable polyphenols in both farming systems.
\end{abstract}

Keywords: Passiflora edulis Sims; management; soluble solids; phenolic compounds.

\section{INTRODUÇÃO}

A agricultura orgânica baseia-se no equilíbrio ecológico da unidade de produção, a fim de privilegiar o desenvolvimento sustentável dos agroecossistemas. O sistema de manejo orgânico atua favorecendo a biodiversidade, aos ciclos biológicos e atividade biológica do solo utilizando fertilizantes orgânicos e fontes de energia renováveis. No entanto, ainda é crescente o uso indiscriminado de pesticidas químicos sintéticos em cultivos convencionais, o que tem contribuído para o desequilíbrio ambiental e provocados inúmeros prejuízos a saúde de trabalhadores de campo e consumidores. Atualmente, os consumidores estão cada vez mais preocupados com o bemestar e saúde. Aliado a estes aspectos, é importante ampliar a demanda por alimentos orgânicos que atendam às exigências dos consumidores em relação à qualidade, segurança alimentar e proteção ambiental (MAGKOS; ARVANITI; ZAMPELAS, 2006; ANASTACIA, 2021).

Ao longo dos anos diferentes alimentos têm sido produzidos pela agricultura orgânica. Aparentemente, há uma percepção geral na população de que alimentos orgânicos são mais saudáveis, saborosos e nutritivos do que os alimentos produzidos convencionalmente. Entretanto, a evidência científica é insuficiente para confirmar ou rejeitar essa suposição, uma vez que dados comparativos dos dois sistemas de produção são inadequados ou inconsistentes devido à heterogeneidade do material e metodologias de pesquisas utilizadas (WILLER; SCHAACK; LERNOUD, 2018). 
O maracujá é uma frutífera pertencente à família Passifloraceae. Originária da América Tropical, a espécie tem sido relatada como de grande expressão socioeconômica no mundo. Aproximadamente 805 mil toneladas do fruto foram produzidas no mundo em 2019, destacando como países de maior produção o Brasil, Vietnã e Colômbia. A região Nordeste é responsável pela maior produção dos frutos de maracujá no país (ALTENDORF, 2018; IBGE, 2020).

No mundo, são identificadas mais de 500 espécies do gênero Passiflora, distribuídas principalmente em regiões tropicais e subtropicais, sendo cerca de 150 espécies encontradas no Brasil. Das espécies já descritas, Passiflora edulis fo. edulis Sims, Passiflora alata, Passiflora ligularis e Passiflora quadrangularis são as mais difundidas e cultivadas comercialmente (PEREIRA; CORREA; OLIVEIRA, 2015; CERQUEIRA-SILVA et al., 2016).

Entre as espécies de maracujá cultivadas no Brasil, encontra-se o maracujá-roxo (Passiflora edulis Sims), com produção de frutos que podem ser aproveitados como alimento. Os frutos de maracujá-roxo são do tipo ovoide, apresentando grandes variações no comprimento e largura. A cor da casca é totalmente arroxeada e da polpa amarela escura. As sementes são ovais providas de arilo membranoso e endosperma carnoso (THOKCHOM; MANDAL, 2017). O fruto é de grande utilidade, principalmente quando consumido na forma in natura ou destinado à produção de suco concentrado, sendo as cascas e sementes, resultantes de seu processamento, os principais co-produtos. As características de alto rendimento em polpa, elevado teor de sólidos solúveis e compostos bioativos presentes nos frutos, são bem importantes, principalmente pela contribuição dos efeitos benéficos que estes podem proporcionar a saúde humana e estarem relacionados à redução do risco e desenvolvimento de doenças (RAMAIYA et al., 2019).

O estudo de maracujá-roxo torna-se interessante devido ainda as poucas informações literárias sobre a determinação da composição nutricional e bioativa, os atributos de qualidade à comercialização e utilização deste fruto na elaboração de produtos industrializados (THOKCHOM; MANDAL, 2017; REIS et al., 2018; RAMAIYA et al., 2019), no entanto, uma caracterização detalhada incluindo seus coprodutos, como cascas, bagaços e compostos com propriedades funcionais individuais nos frutos ainda não bem foi relatada até o momento, o que merece mais atenção e novos estudos devem ser conduzidos e publicados por parte dos pesquisadores científicos a nível mundial.

Sendo assim, o presente trabalho teve por objetivo determinar as características físico-químicas, centesimais, compostos bioativos e capacidade antioxidante dos frutos de maracujá-roxo, cultivados em manejo convencional e orgânico.

\section{MATERIAL E MÉTODOS}

Foram utilizados frutos de maracujá-roxo, híbrido intraespecífico de Passiflora edulis Sims, da série HFOP - 06, oriundo de cruzamentos entre parentais selecionados pela Embrapa Mandioca e Fruticultura e provenientes de sistema de cultivo convencional e orgânico. Os frutos para estudo foram coletados de 100 plantas em 2018 na Estação Experimental Fazendinha Agroecológica localizada em Seropédica - RJ (Latitude: -22.7414, Longitude: -43.7053, $22^{\circ}$ 44' 29" Sul, 43 42' 19" Oeste) e altitude em torno de $26 \mathrm{~m}$. O clima, segundo Köppen, é do tipo Aw, com verões chuvosos e invernos secos. As médias mensais da temperatura mais baixa e mais alta são $20{ }^{\circ} \mathrm{C}$ e $29{ }^{\circ} \mathrm{C}$, respectivamente, com precipitação anual em torno de 1.250 $\mathrm{mm}$.

O solo da área experimental é classificado como latossolo vermelho, distrófico e textura argilo-arenosa. Os maracujazeiros no sistema convencional e orgânico foram adubados com base na análise química do solo, conforme as recomendações para a cultura. Antes do plantio, as covas $(0,4$ x $0,4 \times 0,4 \mathrm{~m}$ ) foram abertas e adubadas com $5 \mathrm{~L}$ de esterco de composto orgânico, $0,3 \mathrm{~kg}$ de termofosfato $(17,5 \%$ de $\left.\mathrm{P}_{2} \mathrm{O}_{5}\right), 100 \mathrm{~g}$ de gesso agrícola, $100 \mathrm{~g}$ de calcário dolomítico, em ambos os sistemas de cultivo. As fontes fertilizantes utilizadas foram torta de mamona $(5,5 \%$ de $\mathrm{N})$ e sulfato de potássio $\left(50 \%\right.$ de $\left.\mathrm{K}_{2} \mathrm{O}\right)$ para cultivo em manejo orgânico e sulfato de amônio $(20 \%$ de $\mathrm{N}$ ) e sulfato de potássio $(50 \%$ de $\left.\mathrm{K}_{2} \mathrm{O}\right)$ para cultivo convencional. Em ambos os cultivos, adotou-se o espaçamento de 2,5 $\mathrm{m}$ entre linhas e 2,0 $\mathrm{m}$ entre plantas (2000 plantas ha-1), com suprimento de irrigação por gotejamento, onde os tratamentos foram os seguintes volumes de água: 610,8; 1018,1; 1527,1; 2036,1; 3054,2 L.planta ${ }^{-1}$.

As plantas foram conduzidas com apenas uma haste principal, procedendo-se podas das brotações laterais do nível do solo até a altura do arame. Os ramos que se desenvolveram acima do fio de arame foram mantidos, a fim de formar a cortina de produção, sendo sempre podados a uma distância aproximada de $15 \mathrm{~cm}$ do solo, evitando o contato dos ramos com a terra e entrada de possíveis patógenos.

Para o controle de pragas, foi priorizado o controle biológico, com a aplicação de tricoderma. O maior problema fitossanitário observado foi o ataque das lagartas desfolhadoras, que eram retiradas manualmente, além de percevejos e antracnose, mas com baixa infestação e menor dano observado. O controle do mato nas entrelinhas foi realizado com o auxílio de roçadeiras mecânicas e capina manual, nas primeiras semanas, do mato desenvolvido na área da cova descoberta pela ráfia.

Os frutos foram colhidos em estágio de maturação comercial (maduro) (THOKCHOM; MANDAL, 2017) (Figura 1), nas primeiras horas do dia. Em seguida, selecionados, embalados em sacos plásticos e encaminhados ao Laboratório de Alimentos e Bebidas (LAAB) do Instituto de Tecnologia da Universidade Federal Rural do Rio de Janeiro em Seropédica - RJ.

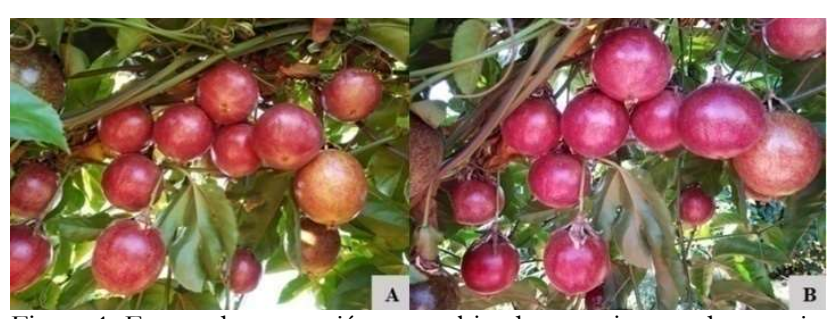

Figura 1. Frutos de maracujá-roxo cultivados em sistema de manejo convencional (A) e orgânico (B). (Fonte: autores).

Figure 1. Purple passion fruits cultivated in conventional (A) and organic (B) management systems. (Source: authors).

Para as avaliações físicas foram tomados vinte frutos e realizadas mensurações individuais para cada fruto estudado. Em seguida, foi realizado o processamento da polpa por meio de multiprocessador WALITA ${ }^{\circledR}$, estabelecendo três 
repetições para a caracterização dos frutos. Os frutos foram analisados quanto a:

Características físicas: Massa fresca dos frutos (g): obtido mediante leitura direta em balança semi-analítica (Marca Marte AD 200); Diâmetro longitudinal e transversal $(\mathrm{mm})$ : determinados com auxílio de paquímetro digital (Marca: Digimess; Modelo: 100.174BL); DL/DT: o índice de formato do fruto foi estabelecido a partir da razão entre o diâmetro longitudinal e transversal; Espessura da casca $(\mathrm{mm})$ : determinados com auxílio de paquímetro digital (Marca: Digimess; Modelo: 100.174BL); Firmeza da casca $(\mathrm{N})$ : com auxílio de um penetrômetro manual Magness Taylor modelo FT 011 com ponteira de $12 \mathrm{~mm}$ de diâmetro. Foram realizadas três leituras em lados opostos da porção equatorial de cada fruto, obtendo-se a média e rendimento das partes constituintes dos frutos (\%).

Composição centesimal: Umidade (\%): utilizou-se o método gravimétrico, através da perda de peso da amostra submetida a aquecimento em estufa a $105^{\circ} \mathrm{C}$, até a obtenção de peso constante do produto dessecado (AOAC, 2016); Proteínas (\%): determinada pelo método de Kjeldahl (AOAC, 2016); Lipídeos (\%): a determinação de lipídeos foi feita conforme método de Soxhlet (AOAC, 2016); Cinzas (\%): as cinzas foram determinadas por incineração do material em mufla a $550{ }^{\circ} \mathrm{C}$ (AOAC, 2016); Carboidratos (\%): o conteúdo de carboidratos foi determinado por diferença. Calculou-se a média da porcentagem de umidade, proteínas, lipídeos e cinzas e o restante foi considerado carboidrato (AOAC, 2016) e valor energético $(\mathrm{kcal} / 100 \mathrm{~g})=[($ proteínas $\mathrm{x} 4 \mathrm{kcal} / \mathrm{g})+$ (carboidratos x $4 \mathrm{kcal} / \mathrm{g})+$ (lipídeos x $9 \mathrm{kcal} / \mathrm{g})]$ x 100 (AOAC, 2016).

Características físico-químicas: Sólidos solúveis ( ${ }^{\circ}$ Brix): determinados com auxílio de refratômetro digital da marca ATAGO PR-101 (0 a $45^{\circ}$ Brix) (AOAC, 2016); Acidez titulável (\%): determinada por titulometria com $\mathrm{NaOH}(0,1$ $\mathrm{N}$ ), sendo os resultados expressos em porcentagem de ácido cítrico (AOAC, 2016); SS/AT: obtida mediante o quociente entre as duas características e $\mathrm{pH}$ : determinado com auxílio de potenciômetro digital (Mettler DL 12) com membrana de vidro, calibrado com tampões de pH 4 e 7 (AOAC, 2016).

Compostos bioativos: Ácido ascórbico (mg/100 g): teor de ácido ascórbico por titulação com solução de DFI (2,6dicloro-fenol-indofenol 0,02\%), de acordo com Dinesh et al. (2015); Carotenoides totais (mg/100 g): determinados pelo método de Higby (1962), com modificações; Antocianinas totais e flavonoides amarelos $(\mathrm{mg} / 100 \mathrm{~g})$ : segundo Francis (1982); Clorofila total da casca (mg/100 g): determinada conforme recomendação de Bruinsma (1963) e calculada pela equação de Engel e Poggiani (1991) e polifenóis extraíveis totais (mg GAE/100 g): segundo Swain e Hillis (1959), com modificações.

Capacidade antioxidante: DPPH ( $\mu \mathrm{mol}$ TE/100 g): o ensaio de eliminação de radicais livres de DPPH foi medido usando o teste de radicais livres de DPPH (2,2-difenil-1picril-hidrazil), empregando o método de Rufino et al. (2010); FRAP ( $\mu \mathrm{mol}$ TE/100 g): a capacidade antioxidante de cada amostra foi estimada pelo ensaio FRAP, seguindo o procedimento descrito na literatura por Thaipong et al. (2006).

Os resultados foram submetidos à análise de variância constante (homocedasticidade) (ANOVA) e as médias dos dados foram comparadas pelo teste de Tukey, a 5\% de probabilidade. As análises foram realizadas pelo sistema de análise de variância de programa computacional - SISVAR (FERREIRA, 2014).

\section{RESULTADOS}

A caracterização física dos frutos de maracujá-roxo cultivados em sistema convencional e orgânico encontra-se na Tabela 1.

Tabela 1. Características físicas dos frutos de maracujá-roxo em sistemas de cultivo convencional e orgânico.

Table 1. Physical characteristics of purple passion fruits in conventional and organic cultivation systems.

\begin{tabular}{cllll}
\hline Variáveis & \multicolumn{1}{c}{$\mathrm{C}^{7}$} & \multicolumn{1}{c}{$\mathrm{O}^{8}$} & $\mathrm{MG}^{9}$ & $\mathrm{CV}^{10}$ \\
\hline $\mathrm{MF}^{1}(\mathrm{~g})$ & $169,94 \pm 2,21^{\mathrm{a}}$ & $148,20 \pm 2,09^{\mathrm{b}}$ & 159,07 & 24,45 \\
$\mathrm{DL}^{2}(\mathrm{~mm})$ & $75,75 \pm 0,54^{\mathrm{b}}$ & $76,90 \pm 1,12^{\mathrm{a}}$ & 76,33 & 8,16 \\
$\mathrm{DT}^{3}(\mathrm{~mm})$ & $81,75 \pm 0,62^{\mathrm{a}}$ & $77,52 \pm 0,89^{\mathrm{b}}$ & 79,63 & 8,15 \\
$\mathrm{IF}^{4}$ & $0,93 \pm 0,04^{\mathrm{b}}$ & $0,99 \pm 0,03^{\mathrm{a}}$ & 0,96 & 7,96 \\
$\mathrm{EC}^{5}(\mathrm{~mm})$ & $5,59 \pm 0,22^{\mathrm{b}}$ & $6,35 \pm 0,23^{\mathrm{a}}$ & 5,97 & 7,68 \\
$\mathrm{FC}^{6}(\mathrm{~N})$ & $79,47 \pm 2,20^{\mathrm{b}}$ & $96,76 \pm 0,98^{\mathrm{a}}$ & 88,11 & 13,61 \\
\hline
\end{tabular}

${ }^{1}$ Massa fresca; ${ }^{2}$ Diâmetro longitudinal; ${ }^{3}$ Diâmetro transversal; ${ }^{4}$ Índice de formato (DL/DT); ${ }^{5}$ Espessura da casca; ${ }^{6}$ Firmeza da casca; ${ }^{7}$ Convencional; ${ }^{8}$ Orgânico; ${ }^{9}$ Média geral; ${ }^{10}$ Coeficiente de variação. Médias seguidas de mesma letra nas colunas não diferem, segundo o teste de Tukey, ao nível de $5 \%$ de probabilidade.

Os métodos de cultivo (convencional e orgânico) apresentaram diferenças em todas as características físicas avaliadas. Observou-se maior massa média de frutos de maracujá-roxo cultivados em sistema convencional, valor este de 169,94 g. Os frutos de maracujá-roxo cultivados em sistema orgânico e convencional apresentaram os maiores diâmetros longitudinais e transversais, com valores de 76,90 e $81,75 \mathrm{~mm}$, respectivamente. Houve variação quanto aos índices médios de formato do fruto. Para espessura da casca dos frutos, foram observadas diferenças quanto aos sistemas de cultivo utilizados. Pela firmeza externa (casca), os resultados mostram que os frutos de maracujá-roxo cultivados em sistema orgânico obtiveram o maior valor médio $(96,76 \mathrm{~N})$.

O rendimento das partes constituintes dos frutos de maracujá-roxo cultivados em sistema convencional e orgânico é apresentado na Tabela 2.

Tabela 2. Constituição percentual das partes dos frutos de maracujároxo em sistemas de cultivo convencional e orgânico.

Table 2. Percentage constitution of parts of purple passion fruits in conventional and organic cultivation systems.

\begin{tabular}{ccc}
\hline \multirow{2}{*}{ Partes } & \multicolumn{2}{c}{$*$ Constituição percentual (\%) } \\
& Convencional & Orgânico \\
\hline $\begin{array}{c}\text { Polpa (suco }+ \\
\text { semente) }\end{array}$ & $49,04 \pm 2,64$ & $45,94 \pm 2,54$ \\
Casca & $50,96 \pm 2,80$ & $54,06 \pm 3,71$ \\
\hline
\end{tabular}

*Valores médios \pm desvio padrão determinados com base na média de 20 frutos.

O rendimento de polpa (suco + semente) foi de 49,04 e 45,94 , respectivamente, para o cultivo convencional e orgânico. Além disto, deve-se ressaltar o bom rendimento da casca em ambos os sistemas de cultivo.

A composição centesimal dos frutos de maracujá-roxo cultivados em sistema convencional e orgânico é apresentada na Tabela 3. O conteúdo aproximado nas polpas dos frutos de maracujá-roxo foi representado categoricamente como umidade $>$ carboidratos $>$ proteínas $>$ cinzas $>$ lipídeos. Foram observadas diferenças significativas quanto aos valores médios para a composição centesimal dos frutos de maracujá 
em ambos os sistemas de cultivo, exceto para umidade. $\mathrm{O}$ valor energético da polpa nos frutos de maracujá-roxo estudados foi de $43,81 \mathrm{kcal} / 100 \mathrm{~g}$.

As características físico-químicas dos frutos de maracujároxo cultivados em sistema convencional e orgânico encontram-se na Tabela 4.

Tabela 3. Composição centesimal dos frutos de maracujá-roxo em sistemas de cultivo convencional e orgânico.

Table 3. Proximate composition of purple passion fruits in conventional and organic cultivation systems.

\begin{tabular}{ccccc}
\hline Compostos & $\mathrm{C}^{7}$ & $\mathrm{O}^{8}$ & $\mathrm{MG}^{9}$ & $\mathrm{CV}^{10}$ \\
\hline $\mathrm{UMI}^{1}(\%)$ & $89,38 \pm 0,04^{\mathrm{a}}$ & $89,11 \pm 0,09^{\mathrm{a}}$ & 89,25 & 0,05 \\
$\operatorname{PROT}^{2}(\%)$ & $1,25 \pm 0,01^{\mathrm{b}}$ & $1,40 \pm 0,04^{\mathrm{a}}$ & 1,33 & 4,30 \\
$\mathrm{LIP}^{3}(\%)$ & $0,26 \pm 0,02^{\mathrm{b}}$ & $0,38 \pm 0,04^{\mathrm{a}}$ & 0,32 & 16,41 \\
$\mathrm{CINZ}^{4}(\%)$ & $0,73 \pm 0,04^{\mathrm{a}}$ & $0,55 \pm 0,01^{\mathrm{b}}$ & 0,64 & 7,69 \\
$\mathrm{CAR}^{5}(\%)$ & $8,36 \pm 0,04^{\mathrm{b}}$ & $8,54 \pm 0,04^{\mathrm{a}}$ & 8,45 & 0,38 \\
$\mathrm{VCE}^{6}(\mathrm{kcal} / 100)$ & $40,86 \pm 0,23^{\mathrm{b}}$ & $45,95 \pm 3,61^{\mathrm{a}}$ & 43,81 & 8,27
\end{tabular}

${ }^{1}$ Umidade; ${ }^{2}$ Proteínas; ${ }^{3}$ Lipídeos; ${ }^{4}$ Cinzas; ${ }^{5}$ Carboidratos; ${ }^{6}$ Valor calórico energético; ${ }^{7}$ Convencional; ${ }^{8}$ Orgânico; ${ }^{9}$ Média geral; ${ }^{10}$ Coeficiente de variação. Médias seguidas de mesma letra nas colunas não diferem, segundo o teste de Tukey, ao nível de $5 \%$ de probabilidade.

Tabela 4. Características físico-químicas dos frutos de maracujároxo em sistemas de cultivo convencional e orgânico.

Table 4. Physicochemical characteristics of purple passion fruits in conventional and organic cultivation systems.

\begin{tabular}{ccccc}
\hline Frutos & $\mathrm{SS}^{1}\left({ }^{\circ}\right.$ Brix $)$ & $\mathrm{AT}^{2}(\%)$ & $\mathrm{SS} / \mathrm{AT}^{3}$ & $\mathrm{pH}^{4}$ \\
\hline $\mathrm{C}^{5}$ & $13,86 \pm 0,04^{\mathrm{a}}$ & $4,62 \pm 0,01^{\mathrm{a}}$ & $2,99 \pm 0,01^{\mathrm{a}}$ & $2,46 \pm 0,04 \mathrm{~b}$ \\
$\mathrm{O}^{6}$ & $12,93 \pm 0,04 \mathrm{~b}$ & $4,43 \pm 0,02^{\mathrm{b}}$ & $2,94 \pm 0,03^{\mathrm{a}}$ & $2,66 \pm 0,04 \mathrm{a}$ \\
$\mathrm{MG}^{7}$ & 13,40 & 4,52 & 2,96 & 2,56 \\
$\mathrm{CV}^{8}$ & 0,61 & 0,74 & 2,34 & 2,75 \\
\hline${ }^{1}$ Sólidos $^{2}$ & & &
\end{tabular}

${ }^{1}$ Sólidos solúveis; ${ }^{2}$ Acidez titulável; ${ }^{3}$ Relação $\quad$ SS/AT; ${ }^{4}$ Potencia hidrogênionico. ${ }^{5}$ Convencional; ${ }^{6}$ Orgânico; ${ }^{7}$ Média geral; ${ }^{8}$ Coeficiente de variação. Médias seguidas de mesma letra nas linhas não diferem, segundo o teste de Tukey, ao nível de 5\% de probabilidade.

Foram observadas diferenças nos teores médios de sólidos solúveis dos frutos de maracujá-roxo cultivados em manejo convencional e orgânico. Para a acidez total titulável, observaram-se diferenças entre os valores médios dos frutos de maracujá-roxo cultivados em manejo convencional e orgânico. Não houve diferença quanto aos valores médios obtidos para a relação SS/AT. O pH dos frutos de maracujároxo apresentaram valores médios de 2,66 e 2,46 para os sistemas de cultivo orgânico e convencional, respectivamente.

Os compostos bioativos dos frutos de maracujá-roxo cultivados em sistema convencional e orgânico encontram-se na Tabela 5 .

Para o ácido ascórbico, os frutos de maracujá-roxo cultivados em sistema convencional destacaram-se por apresentar um teor médio de 67,08 mg/100 g. Para a análise de carotenoides totais, observaram-se diferenças nos teores médios entre os frutos de maracujá-roxo cultivados em sistema convencional e orgânico, tendo o sistema de manejo convencional obtido o maior valor médio. Os frutos de maracujá-roxo cultivados em sistema orgânico apresentaram os maiores teores médios para flavonoides amarelos (5,57 $\mathrm{mg} / 100 \mathrm{~g})$, entretanto, os frutos de maracujá-roxo cultivados em sistema convencional, destacaram-se no conteúdo de antocianinas totais, apresentando valor médio de 0,52 $\mathrm{mg} / 100 \mathrm{~g}$. Houve diferenças quanto aos teores médios de clorofila total da casca em frutos de maracujá-roxo cultivados em sistema convencional e orgânico. Para os polifenóis extraíveis totais houve diferenças quanto aos teores médios dos frutos de maracujá-roxo cultivados em sistema convencional e orgânico. O sistema de manejo convencional apresentou um teor médio de 16,81 mg GAE/100 g, resultado numericamente mais expressivo quando comparado ao sistema orgânico.

A Tabela 6 apresenta a capacidade antioxidante dos frutos de maracujá-roxo cultivados em sistema convencional e orgânico.

Tabela 5. Compostos bioativos dos frutos de maracujá-roxo em sistemas de cultivo convencional e orgânico.

Table 5. Bioactive compounds of purple passion fruits in conventional and organic cultivation systems.

\begin{tabular}{ccccc}
\hline $\begin{array}{c}\text { Compostos } \\
(\mathrm{mg} / 100 \mathrm{~g})\end{array}$ & $\mathrm{C}^{7}$ & $\mathrm{O}^{8}$ & $\mathrm{MG}^{9}$ & $\mathrm{CV}^{10}$ \\
\hline $\mathrm{AA}^{1}$ & $67,08 \pm 1,27^{\mathrm{a}}$ & $61,85 \pm 2,46^{\mathrm{b}}$ & 64,46 & 1,81 \\
$\mathrm{CT}^{2}$ & $2,74 \pm 0,03^{\mathrm{a}}$ & $1,68 \pm 0,02^{\mathrm{b}}$ & 2,21 & 1,46 \\
$\mathrm{FA}^{3}$ & $4,58 \pm 0,34^{\mathrm{b}}$ & $5,57 \pm 0,04^{\mathrm{a}}$ & 5,07 & 7,08 \\
$\mathrm{ANT}^{4}$ & $0,52 \pm 0,25^{\mathrm{a}}$ & $0,41 \pm 0,02^{\mathrm{b}}$ & 0,46 & 36,18 \\
$\mathrm{CLTC}^{5}$ & $22,49 \pm 2,32^{\mathrm{a}}$ & $21,33 \pm 2,22^{\mathrm{b}}$ & 21,91 & 8,04 \\
$\mathrm{PET}^{6}$ & $16,81 \pm 2,65^{\mathrm{a}}$ & $14,68 \pm 0,58^{\mathrm{b}}$ & 15,74 & 10,47 \\
\hline${ }^{1}$ Ácido ascórbico; ${ }^{2}$ Carotenoides & totais; & ${ }^{3} \mathrm{~F}$ lavonoides & amarelos; \\
${ }^{4}$ Antocianinas totais; ${ }^{5}$ Clorofila total da Casca; ${ }^{\circ}$ Polifenóis extraíveis totais; \\
${ }^{7}$ Convencional; ${ }^{8}$ Orgânico; ${ }^{9}$ Média geral; ${ }^{10} \mathrm{Coeficiente} \mathrm{de} \mathrm{variação.} \mathrm{Médias}$ \\
seguidas de mesma letra nas colunas não diferem, segundo o teste de Tukey, \\
ao nível de 5\% de probabilidade.
\end{tabular}

ao nível de $5 \%$ de probabilidade.

Tabela 6. Capacidade antioxidante dos frutos de maracujá-roxo em sistemas de cultivo convencional e orgânico.

Table 6. Antioxidant capacity of purple passion fruits in conventional and organic cultivation systems.

\begin{tabular}{ccc}
\hline \multirow{2}{*}{ Frutos } & \multicolumn{2}{c}{$\begin{array}{c}\text { Capacidade antioxidante } \\
(\mu \mathrm{mol} \mathrm{TE} / 100 \mathrm{~g})\end{array}$} \\
\cline { 2 - 3 } & $\mathrm{DPPH}^{1}$ & FRAP $^{2}$ \\
\hline $\mathrm{C}^{3}$ & $31,47 \pm 1,15^{\mathrm{b}}$ & $70,05 \pm 1,77 \mathrm{a}$ \\
$\mathrm{O}^{4}$ & $35,86 \pm 1,29 \mathrm{a}$ & $34,53 \pm 9,79 \mathrm{~b}$ \\
$\mathrm{MG}^{5}$ & 33,66 & 52,29 \\
$\mathrm{CV}^{6}$ & 3,98 & 11,12 \\
\hline
\end{tabular}

12,2-difenil-1-picrilhidrazil; ${ }^{2}$ Poder antioxidante de redução do ferro. ${ }^{3}$ Convencional; ${ }^{4}$ Orgânico; ${ }^{5}$ Média geral. ${ }^{6}$ Coeficiente de variação. Médias seguidas de mesma letra nas linhas não diferem, segundo o teste de Tukey, ao nível de $5 \%$ de probabilidade.

Para a capacidade antioxidante dos frutos de maracujároxo cultivados em sistemas convencional e orgânico houve variação nos teores médios quando da determinação pelos métodos de avaliação DDPH e FRAP.

\section{DISCUSSÃO}

Negreiros et al. (2008) avaliaram a relação entre as características físicas e o rendimento de polpa de maracujáamarelo em cultivo convencional e observaram para a massa fresca dos frutos valores médios próximos ao do referido estudo em ambos os sistemas de cultivo. Hurtado-Salazar et al. (2015) avaliaram as massas frescas dos frutos de maracujáamarelo enxertado em espécies silvestres do gênero passiflora cultivado em ambiente protegido e observaram para a massa fresca dos frutos valores médios inferiores ao do referido estudo em ambos os sistemas de cultivo. A massa fresca dos frutos é uma característica que está relacionada linearmente com o seu grau de desenvolvimento ou maturação. O aumento gradativo da massa fresca de frutos durante o seu desenvolvimento ocorre devido à maior quantidade de fotoassimilados, açúcares e carboidratos acumulados 
(TESSMER et al., 2014). Além disto, o peso médio de frutos é uma característica importante para o mercado in natura, sendo mais valorizados os frutos com maior peso. No Estado do Rio de Janeiro a produção de maracujá é destinada em sua maior parte para o mercado in natura e essa característica deve ser um importante critério de seleção de variedades para o plantio.

Negreiros et al. (2008) estudaram o diâmetro longitudinal e transversal em frutos de maracujá-amarelo e encontraram resultados médios próximos ao do referido estudo. HurtadoSalazar et al. (2015) estudaram o diâmetro longitudinal e transversal em frutos de maracujá-amarelo enxertado em espécies silvestres do gênero passiflora cultivado em ambiente protegido e encontraram resultados médios de 89,05 e 70,02 mm, respectivamente. Essas características tornam-se extremamente desejáveis, uma vez que os frutos de maracujá são classificados comercialmente em relação à medida de seu diâmetro. No que se diz respeito ao índice de formato do fruto, esse é um parâmetro físico importante para frutos destinados ao mercado in natura. Valores próximos de 1,0 indicam que os frutos possuem formatos mais arredondados e acima formatos oblongos (GRECO et al., 2014).

Negreiros et al. (2008) e Hurtado-Salazar et al. (2015) avaliaram as espessuras das cascas em frutos de maracujáamarelo e encontraram resultados na mesma faixa. De acordo com Freire et al. (2014a) e Freire et al. (2014b) frutos com casca fina são mais desejáveis pelo consumidor, uma vez que para a comercialização apresentam maior rendimento de polpa por quilograma adquirido.

Dias et al. (2012) avaliaram a firmeza em frutos de maracujá-amarelo submetidos a diferentes condutividades e frequências elétricas da água de irrigação com aplicação de biofertilizantes. A firmeza é uma característica de qualidade relacionada à maturação dos frutos e ponderada pelo consumidor, sendo que à medida que avança o processo de maturação dos mesmos eles tendem a ficar menos firmes, com conseqüente redução da sua vida útil pós-colheita e aceitabilidade.

Freire et al. (2014a) e Greco et al. (2014) avaliaram o rendimento em polpas de frutos de maracujá-amarelo e azedo. De acordo com Negreiros et al. (2008) o rendimento de polpa considerado como adequados tanto para o consumo in natura como para a agroindústria em frutos de maracujá deve ser superior a $50 \%$. Os mesmos autores concluíram que a seleção dos frutos com maior diâmetro equatorial possibilita a obtenção de maracujás mais pesados e com maior rendimento de polpa, evidenciado pelo maior efeito direto do diâmetro sobre o peso da polpa e rendimento. Também concluíram que o rendimento da polpa pode ser selecionado indiretamente, com base na menor espessura da casca e que não foi observada correlação entre relação comprimento/diâmetro (maior em frutos ovais) e rendimento de polpa.

Algumas pesquisas relacionadas com a composição centesimal têm sido realizadas com espécies de maracujá em diferentes sistemas de cultivo, indicando que o consumo do fruto de maracujá-roxo no estágio maduro pode ser realizado a fim de suprir as necessidades energéticas diárias (GRECO et al., 2014; REIS et al., 2018; RAMAIYA et al., 2019). Os níveis de umidade encontrados nos frutos de maracujá-roxo foram comparativamente mais altos, o que os indicou como uma boa fonte de meio para o funcionamento de enzimas e processos metabólicos gerais. O teor de umidade também é importante para a estabilidade e a qualidade dos sucos. Amostras de suco com alta porcentagem de cinzas implicam que os frutos contêm altas concentrações de vários conteúdos minerais, os quais são antecipados para acelerar as ações metabólicas e melhorar o crescimento e desenvolvimento. Sucos de maracujás com poucos carboidratos podem ser ideais para pacientes diabéticos e hipertensos que necessitam de dietas com pouco açúcar (RAMAIYA et al., 2019). A ingestão adequada destes componentes, inclusive de fibras alimentares pode reduzir o risco de doenças cardíacas, colesterol, constipação, hipertensão, diabetes e cânceres (REIS et al., 2018).

Freire et al. (2014b) avaliaram os teores de sólidos solúveis em sucos dos frutos de maracujá-amarelo. Greco et al. (2014) avaliaram os teores de sólidos solúveis em polpas dos frutos de maracujá-azedo. Hurtado-Salazar et al. (2015) avaliaram os teores de sólidos solúveis em polpas de frutos de maracujá-roxo e encontraram valor médio de $12,65^{\circ}$ Brix, bem inferior ao do referido estudo. Entretanto, Reis et al. (2018) analisando esta característica em polpas de frutos de maracujá-roxo encontraram valores bem abaixos. Valores em torno de $13,0{ }^{\circ}$ Brix são desejáveis pelas indústrias de processamento de polpas de maracujá, pois permitem um melhor desempenho tecnológico (MANIWARA et al., 2014). Cabe ressaltar ainda que por ser uma característica de relevante importância, a mesma é muito influenciada por fatores do meio, entre os quais se destacam o local de plantio e condução da cultura.

Freire et al. (2014b) e Greco et al. (2014) avaliaram os teores de acidez titulável em polpas dos frutos de maracujáamarelo e azedo. Negreiros et al. (2008) e Reis et al. (2018) encontraram teores médios de acidez de 3,98\% e 2,83\% em frutos de maracujá-amarelo e maracujá-roxo, respectivamente. De acordo com Negreiros et al. (2008) a acidez em frutos é uma característica também importante para a indústria, pois desfavorece a manifestação de microrganismos e, conseqüentemente, confere maior tempo de conservação do produto. Por outro lado, a acidez proporciona um sabor não muito doce, o que não agrada aos consumidores de frutas in natura. Tendo em vista as modalidades de mercado a que se destinam, os frutos apresentaram características de acidez totais adequadas tanto para frutos in natura quanto para industrialização.

Negreiros et al. (2008) e Hurtado-Salazar et al. (2015) avaliaram a SS/AT de frutos de maracujá-amarelo e encontraram valor médio bem próximo aos reportados neste trabalho. Greco et al. (2014) avaliaram a SS/AT de frutos de maracujá-azedo. A relação SS/AT é considerada uma das formas mais práticas de avaliar-se o sabor dos frutos. O teor de açúcar e acidez dos frutos pode sofrer variação em decorrência de fatores ambientais e práticas de cultivo, qualidade de luz solar e temperatura, como também do tipo e dosagens de fertilizantes; portanto, com reflexos diretos na relação SS/AT. Esta relação fornece o indicativo do sabor dos frutos, pois relaciona a quantidade de açúcares e ácidos presentes proporcionando boa ideia do equilíbrio entre esses dois componentes (NEGREIROS et al., 2008).

Freire et al. (2014b) e Greco et al. (2014) avaliaram o pH em polpas dos frutos de maracujá-amarelo e azedo. Reis et al. (2018) avaliaram a qualidade pós-colheita de frutos de maracujá e mostram que para o $\mathrm{pH}$ encontrado os valores médios foram bem superiores aos dos reportados neste trabalho. Vale salientar que os fatores ambientais, influenciam 
consideravelmente na composição química dos princípios alimentícios vegetais, onde pode ser esclarecido que a variação na composição química dos frutos deve-se não somente a variedade, mas também ao grau de maturação antes da colheita e as condições de maturação pós-colheita e armazenagem. Desta forma, o valor do $\mathrm{pH}$ pode ser definido como critério de acidez de forma comparativa entre os frutos.

Freire et al. (2014b), Greco et al. (2014), Reis et al. (2018) e Ramaiya et al. (2019) avaliaram os teores de compostos bioativos de frutos de maracujá em diferentes sistemas de cultivo e manejo. O conteúdo de compostos bioativos nos frutos depende diretamente de fatores ambientais como cultivar, região de cultivo e maturação de frutos, e, além disso, o próprio processo de industrialização pode afetar essas características. Os compostos bioativos presentes em frutas, como o ácido ascórbico, carotenoides, flavonoides, antocianinas, clorofilas e polifenóis são ditos como componentes nutricionais importantes e podem ser utilizados como índice de padrão ou de qualidade de alimentos, além de serem compostos voltados a reduzir o risco de doenças (SALEEM et al., 2017).

Estudos recentes têm sido realizados para avaliação da capacidade antioxidante em frutos de espécies de maracujá em diferentes sistemas de cultivo e manejo (REIS et al., 2018; RAMAIYA et al., 2019). Deve-se ressaltar que a capacidade antioxidante em frutas depende de vários fatores, como: estádio de maturação, armazenamento, processamento e preparo. De acordo com pesquisas já realizadas é provado que os radicais livres desempenham papel importante em muitas doenças, como as cardiovasculares e neurodegenerativas, câncer, diabetes e envelhecimento (SALEEM et al., 2017). Antioxidantes têm atraído mais atenção como agentes potenciais para prevenir e tratar o estresse oxidativo relacionados às doenças. Por outro lado, estudos epidemiológicos descobriram que a ingestão de frutas tem forte correlação inversa com o risco de desenvolver muitas doenças crônicas, como doenças cardiovasculares e câncer (NGUYEN et al., 2019).

\section{CONCLUSÕES}

Os frutos analisados apresentaram maiores valores médios para o rendimento de polpa e sólidos solúveis em sistema convencional, que foram de $49,04 \%$ e $13,86^{\circ}$ Brix. O valor calórico energético foi de 45,95 kcal/100 g em sistema orgânico, indicando que o consumo do fruto "maduro" pode ser realizado a fim de suprir as necessidades energéticas diárias.

As polpas dos frutos de maracujá-roxo estudados demonstraram serem fontes importantes de componentes bioativos, com respaldo aos elevados valores médios para ácido ascórbico e polifenóis extraíveis totais em ambos os sistemas de cultivo.

\section{AGRADECIMENTOS}

Os autores agradecem à Universidade Federal Rural do Rio de Janeiro (UFRRJ), Embrapa Agrobiologia e Coordenação de Aperfeiçoamento de Pessoal de Nível Superior (CAPES) pelo apoio e suporte financeiro na execução do presente trabalho.

\section{REFERÊNCIAS}

ALTENDORF, S. Minor tropical fruits: Mainstreaming a niche market. In: FAO (Ed.), Food Outlook. FAO, Rome, Italy, p. 67-74, 2018.

ANASTACIA, C. Systematic literature review on organic farming for sustainable agricultural production. International Journal of Contemporary Research and Review, v. 12, n. 3, p. 20355-20360, 2021. DOI: https://doi.org/10.15520/ijcrr.v12i03.891

AOAC. Association of Agricultural Chemists. Official methods of the association of the agricultural chemists: v. 2. (20rd ed.). Pharmabooks: AOAC International, 2016.

BRUINSMA, J. The quantitative analysis of clorophylls a and $\mathrm{b}$ in plant extracts. Photochemistry and Photobiology, v. 2, n. 2, p. 241-249, 1963. DOI: https://doi.org/10.1111/j.1751-1097.1963.tb08220.x

CERQUEIRA-SILVA, C. S. B. M.; FALEIRO, F. G.; JESUS, O. N.; SANTOS, E. S. L.; SOUZA, A. P. The genetic diversity, conservation and use of passion fruit (Passiflora spp). In: AHUJA, M. R.; JAIN, S. M. (Ed.). Genetic diversity and erosion in plants: case histories. Cham: Springer. il. (Sustainable Development and Biodiversity), v.2, p.215-231, 2016.

DIAS, T. J.; CAVALCANTE, L. F.; NUNES, J. C.; FREIRE, J. L. O.; NASCIMENTO, J. A. M. Qualidade física e produção do maracujá amarelo em solo com biofertilizante irrigado com águas salinas. Semina, v. 33, n. $1, \quad$ p. 2905-2918, 2012. DOI: https://doi.org/10.5433/1679-

0359.2012v33Supl1p2905

DINESH, B.; YADAV, R. B.; REDDY, D. A.; PADMA, S.; SUKUMARAN, M. K. Determination of ascorbic acid content in some Indian spices. International Journal of Current Microbiology and Applied Sciences, v. 4, n. 8, p. 864-868, 2015.

ENGEL, V. L.; POGGIANI, F. Estudo da concentração de clorofila nas folhas e seu espectro de absorção de luz em função do sombreamento em mudas de quatro espécies florestais. Revista Brasileira de Fisiologia Vegetal, v. 3, n. 1, p. 39-45, 1991.

FERREIRA, D. F. Sisvar: a guide for its bootstrap procedures in multiple comparisons. Ciência e Agrotecnologia, v. 38, n. 2, p. 109-112, 2014. DOI: https://doi.org/10.1590/S1413-70542014000200001

FRANCIS, F. J. Analysis of anthocyanins. In: MARKAKIS, P (ed). Anthocyanins as food colors. New York: Academic Press, p. 181-207, 1982.

FREIRE, J. L. O.; CAVALCANTE, L. F.; REBEQUI, A. M.; DIAS, T. J.; BREHM, M. A. S.; SANTOS, J. B. Physical characteristics of fruit of yellow passion fruit produced in soil with bio-fertilizer, mulching and saline water. Idesia, v. 32 , n. 1 , p. 71-78, 2014a. DOI: https://doi.org/10.4067/S0718-34292014000100008

FREIRE, A. L. O.; CAVALCANTE, L. F.; REBEQUI, A. M.; DIAS, T. J.; BREHM, M. A. S; SANTOS, J. B. Qualityofyellowpassionfruitjuicewithcultivationusingdiff erentorganicsourcesand saline water. Idesia, v. 32, n. 1, p. 79-87, 2014b. DOI: https://doi.org/10.4067/S071834292014000100009

GRECO, S. M. L.; PEIXOTO, J. R.; FERREIRA, L. M. Avaliação física, físico-química e estimativas de parâmetros genéticos de 32 genótipos de maracujazeiro- 
azedo cultivados no Distrito Federal. Bioscience Journal, v. 30, n. 1, p. 360-370, 2014.

HIGBY, W. K. A simplifield method for determination of some the carotenoid distribution in natural and carotene fortifield orange juice. Journal of Food Science, v. 27, n. $1, \quad$ p. 42-49, 1962. DOI: https://doi.org/10.1111/j.1365-2621.1962.tb00055.x

HURTADO-SALAZAR, A.; SILVA, D. F. P.; SEDIYAMA, C. S.; BRUCKNER, C. H. Caracterização física e química de frutos de maracujazeiro-amarelo enxertado em espécies silvestres do gênero passiflora cultivado em ambiente protegido. Revista Brasileira de Fruticultura, v. 37, n. 3, p. 635-643, 2015. DOI: https://doi.org/10.1590/0100-2945-101/14

IBGE. Produção agrícola municipal 2020. Disponível em: https://sidra.ibge.gov.br/tabela/5457\#resultado. Acesso em: 09/01/2020. 2020.

MAGKOS, F.; ARVANITI, F.; ZAMPELAS. A. Organic food: buying more safety or just peace of mind? A critical review of the literature. Critical Reviews in Food Science and Nutrition, v. 46, n. 1, p. 23-56, 2006. DOI: https://doi.org/10.1080/10408690490911846

MANIWARA, P.; NAKANO, K.; BOONYAKIAT, D.; OHASHI, S.; HIROI, M.; TOHYAMA, T. The use of visible and near infrared spectroscopy for evaluating passion fruit postharvest quality. Journal of Food Engineering, v. 143, p. 33-43, 2014. DOI: https://doi.org/10.1016/j.jfoodeng.2014.06.028

NEGREIROS, J. R. S.; NETO, S. E. A.; ÁLVARES, V. S.; LIMA, V. A.; OLIVEIRA, T. K. Caracterização de frutos de progênies de meios-irmãos de maracujazeiro-amarelo em Rio Branco - Acre. Revista Brasileira de Fruticultura, v. 30, n. 2, p. 431-437, 2008. DOI: https://doi.org/10.1590/S0100-29452008000200028

NGUYEN, N. M. P.; LE, T. T.; VISSENAEKENS, H.; GONZALES, G. B.; VAN-CAMP, J.; SMAGGHE, G.; RAES, K. In vitro antioxidant activity and phenolic profiles of tropical fruit by-products. International Journal of Food Science and Technology, v. 54, n. 4, p. 1169-1178, 2019. DOI: https://doi.org/10.1111/ijfs.14093

PEREIRA, A. D.; CORREA, R. X.; OLIVEIRA, A. C. Molecular genetic diversity and differentiation of populations of 'somnus' passion fruit trees (Passiflora setacea DC): Implications for conservation and prebreeding. Biochemical Systematics and Ecology, v. 59, n. 1, p. 12-21, 2015. DOI: https://doi.org/10.1016/j.bse.2014.12.020

RAMAIYA, S. D.; BUJANG, J. B.; ZAKARIA, M. H.; SAUPI, N. Nutritional, mineral and organic acid composition of passion fruit (Passiflora species). Food Research, v. 3, n. 3, p. 231-240, 2019. DOI: https://doi.org/10.26656/fr.2017.3(3).233

REIS, L. C. R.; FACCO, E. M. P.; SALVADOR, M.; FLÔRES, S. H.; RIOS, A. O. Antioxidant potential and physicochemical characterization of yellow, purple and orange passion fruit. Journal of Food Science and Technology, v. 55, n. 7, p. 2679-2691, 2018. DOI: https://doi.org/10.1007/s13197-018-3190-2

RUFINO, M. S. M.; ALVES, R. E.; BRITO, E. S.; PÉREZJIMÉNEZ, J.; SAURA CALIXTO, F.; MANCINIFILHO, J. Bioactive compounds and antioxidant capacities of 18 non-traditional tropical fruits from Brazil.
Food Chemistry, v. 121, n. 4, p. 996-1002, 2010. DOI: https://doi.org/10.1016/j.foodchem.2010.01.037

SALEEM, F.; SARKAR, D.; ANKOLEKAR, C.; SHETTY, $K$. Phenolic bioactives and associated antioxidant and anti-hyperglycemic functions of select species of Apiaceae family targeting for type 2 diabetes relevant nutraceuticals. Industrial Crops and Products, v. 107, p. 518-525, 2017.2017 https://doi.org/10.1016/j.indcrop.2017.06.023

SWAIN, T.; HILLIS, W. E. The phenolics constituents of Prunus domestica. The quantitative analysis of phenolic constituents. Journal of the Science of Food and Agriculture, v. 10, n. 1, p. 63-68, 1959. DOI: https://doi.org/10.1002/jsfa.2740100110

TESSMER, M. A.; KLUGE, R. A.; APPEZZATO-DAGLÓRIA, B. The accumulation of tannins during the development of "Giombo" and "Fuyu" persimmon fruits. Scientia Horticulturae, v. 172, p. 292-299, 2014. DOI: https://doi.org/10.1016/j.scienta.2014.04.023

THAIPONG，K.; BOONPRAKOB，U.; CROSBY，K.; CISNEROS-ZEVALLOS, L.; BYRNE, D. H. Comparison of ABTS, DPPH, FRAP and ORAC assays for estimating antioxidant activity from guava fruit extracts. Journal of Food Composition and Analysis, v. 19 , n. 6-7, p. 669-675, 2006. DOI: https://doi.org/10.1016/j.jfca.2006.01.003

THOKCHOM, R.; MANDAL, G. Production preference and importance of passion fruit (Passiflora edulis): A review. Journal of Agricultural Engineering and Food Technology, v. 4, n. 1, p. 27-30, 2017.

WILLER, H.; SCHAACK, D.; LERNOUD, J. Organic farming and market development in Europe and the European Union. In: WILLER, H.; LERNOUD, J. (Eds.). The world of organic agriculture: statistics and emerging trends 2018. Frick: FiBL; Bonn: Ifoam Organics Internacional, 2018. 